\title{
Chromosome 9p21 polymorphism is associated with myocardial infarction but not with clinical outcome in Han Chinese
}

\author{
Wen Hui Peng ${ }^{1,2}$, Lin Lu,2, Qi Zhang1, Rui Yan \\ Zhang ${ }^{1}$, Ling Jie Wang ${ }^{2}$, Xiao Xiang Yan ${ }^{2}$, \\ Qiu Jing Chen ${ }^{2}$ and Wei Feng Shen ${ }^{1,2, *}$ \\ ${ }^{1}$ Department of Cardiology, Rui Jin Hospital, \\ Shanghai Jiaotong University School of Medicine, \\ Shanghai, People's Republic of China \\ ${ }^{2}$ Institute of Cardiovascular Diseases, Shanghai \\ Jiaotong University School of Medicine, Shanghai, \\ People's Republic of China
}

\begin{abstract}
Background: rs1333049 polymorphism on chromosome 9p21 has been shown to affect susceptibility to coronary artery disease (CAD) in Caucasians. This study examined the association of rs1333049 with myocardial infarction (MI), angiographic severity of CAD and clinical outcome after a first acute $\mathrm{MI}$ in Han Chinese.

Methods: rs1333049 polymorphism was genotyped in 520 patients with a first acute $\mathrm{MI}$ and in 560 controls. The number of angiographically documented diseased coronary arteries (luminal diameter stenosis $\geq 50 \%$ ), echocardiographic left ventricular ejection fraction (LVEF), and major adverse cardiac events (MACE) during follow-up (mean, 29 \pm 15 months) were recorded.

Results: Patients with $\mathrm{Ml}$ had higher frequencies of the $C C$ genotype $(30.0 \%$ vs. $20.7 \%$ ) or $C$ allele $(55.5 \%$ vs. $46.2 \%$ ) compared with controls (all $p<0.01$ ). rs1333049 polymorphism was strongly associated with $\mathrm{MI}$ [odds ratio (OR) 1.48, 95\% confidence interval (CI) 1.22-1.79] after adjusting for traditional risk factors. Although longer hospitalization stay was observed in patients with the rs1333049- $C$ allele, this polymorphism was not related to angiographic severity of CAD, LVEF, and occurrence of MACE after MI.

Conclusions: This study demonstrates an association of rs1333049 polymorphism locus on chromosome 9 p21 with risk for $\mathrm{MI}$, but not with post-MI prognosis in Han Chinese.

Clin Chem Lab Med 2009;47:917-22.
\end{abstract}

Keywords: coronary artery disease (CAD); myocardial infarction (MI); outcome; 9p21; polymorphism.

\footnotetext{
*Corresponding author: Wei Feng Shen, PhD, MD, Department of Cardiology, Rui Jin Hospital, 197 Rui Jin Road II, Shanghai 200025, P.R. China Phone: +86-21-64370045, Fax: +86-21-64457177, E-mail: rjshenweifeng@yahoo.com.cn Received February 2, 2009; accepted April 27, 2009; previously published online June 24, 2009
}

\section{Introduction}

It is widely accepted that genetic variation is important for the pathogenesis of coronary artery disease (CAD), myocardial infarction (MI) and clinical outcome $(1,2)$. Although numerous candidate genes have been implicated in the development and progression of atherosclerosis, the genes that are responsible remain largely unknown. Recently, genome-wide association studies have demonstrated a locus on chromosome 9p21 for CAD susceptibility in Caucasians (3-7). Chromosome 9p21 was found to also be associated with other atherosclerotic diseases such as stroke and aneurysm (8-10).

Studies on chromosome 9p21 were performed primarily in Caucasians, while similar studies in Asians are less common (7). As variants and their frequencies of chromosome $9 p 21$ in various ethnic groups might be different, replication is needed to confirm the potential effects of chromosome 9 p21 in other groups. In addition, genetic factors implicated in atherogenesis may affect the outcome of cardiovascular disease. However, little data is available regarding the impact of chromosome 9p21 on the prognosis of Asian patients with CAD. The present study was conducted to assess the association of rs1333049 polymorphism, one representative polymorphism on chromosome 9p21 (7), with $\mathrm{MI}$, angiographic severity of CAD, and occurrence of major adverse cardiac events (MACE) after acute MI in Han Chinese.

\section{Materials and methods}

\section{Study population}

The study protocol was approved by the Hospitals Ethics Committee. All participants were of Chinese Han origin and gave written informed consent. There were 584 patients included in this study who survived a first acute ST-segment elevation MI between November 2003 and December 2006. $\mathrm{Ml}$ was defined as 1 ) ischemic chest pain of $>30 \mathrm{~min}$ duration not relieved by sublingual nitrates, 2) new ST-segment elevation in at least two contiguous leads of 12-lead electrocardiogram, with the cut-off points $\geq 0.2 \mathrm{mV}, 3$ ) new, or presumably new, left bundle branch block (LBBB), 4) increased serum creatine kinase $M B$ and troponin $T$ at least 2 times greater than the upper limit of the reference interval. To avoid confounding results, patients with concomitant severe kidney disease (12 patients), liver disease (13 patients), malignancy ( 2 patients), valvular disease (19 patients) and cardiomyopathy ( 3 patients) were excluded. Fifteen patients lost to follow-up were also excluded leaving 520 patients for inclusion in the analysis. 
The control subjects were residents of three major districts in Shanghai who received an annual physical examination in 2005. A detailed medical and family history was obtained; none had documented CAD, severe kidney or liver disease, or malignancy. The control subjects were selected to match MI patients with respect to age, gender, and traditional risk factors such as smoking, diabetes and hypertension, without knowledge of chromosome 9p21 genotypes.

Coronary angiography was performed using standard Judkins technique or radial approach. Severity of CAD was defined according to the total number of significant stenotic coronary arteries (luminal diameter narrowing $\geq 50 \%$ ). Coronary lesion types $\left(A, B_{1}, B_{2}\right.$, or $\left.C\right)$ were defined according to a modified classification of the American College of Cardiology/ American Heart Association (11).

Two-dimensional echocardiography was performed at discharge using Vivid-7 system (GE Vingmed Sound AS, Horton, Norway). Left ventricular ejection fraction (LVEF) was determined using apical four chamber view by Simpson's method.

Patients with $\mathrm{MI}$ received clinical follow-up in a special outpatient clinic or were contacted by telephone every 3 months after initial angiography (mean, $29 \pm 15$ months). Occurrence of MACE included cardiac death, recurrence of non-fatal $\mathrm{MI}$, recurrent angina or heart failure requiring hospitalization. In order to guarantee quality of the data, cause of death was verified by reviewing hospital records and death certificates. All MACE were reviewed by two experienced interventional cardiologists.

Blood samples were collected after an overnight fast at hospital admission and stored at $-80^{\circ} \mathrm{C}$. White blood cell count, serum glucose, creatinine, and lipid profiles [total cholesterol, low-density lipoprotein cholesterol (LDL-C), high-density lipoprotein cholesterol (HDL-C), lipoprotein (a), apolipoprotein $A$ (apoA), apolipoprotein $B$ (apoB) and triglycerides] were measured (HITACHI 912 Analyzer, Roche Diagnostics, Germany).

\section{Genotype determination}

Whole blood was drawn from each participant and genomic DNA was extracted from peripheral blood leucocytes using standard phenol-chloroform extraction. Genotyping was performed with TaqMan single nucleotide polymorphism (SNP) allelic discrimination by means of an ABI 7900HT (Applied Biosystems, Foster City, CA, USA) in a 384-well format. The TaqMan Assay kit was purchased from Applied Biosystems (Foster City, CA, USA). Genotyping was performed in a $5-\mu \mathrm{L}$ volume containing $2.5 \mu \mathrm{L}$ of TaqMan Universal PCR Master Mix, $0.125 \mu \mathrm{L}$ of $40 \times$ TaqMan MGB Assay Mix, and $25 \mathrm{ng}$ of genomic DNA. Primers of rs1333049 polymorphism were TCACTACCCTACTGTCATTCCTCAT and TTGCfjTTACCTCTGCGAGTGG. Probes were VIC-CAACAGTTCAAAAGCA and FAM-AACAGTTGAAAAGCA. Primers of rs10757274 were CCCCCGTGGGTCAAATCTAAG and AGAATTCCСTACСССTATСTССТАTCT. Probes were VICCTGAGTGTTGAGACATA and FAM-CTGAGTGTTGGGACATA. Data were analyzed using the ABI Prism SDS software version 2.1 (Applied Biosystems).

\section{Statistical analysis}

Continuous and categorical variables were expressed as mean $\pm S D$ and frequencies, respectively. Differences among groups were assessed using the $\chi^{2}$-test, Kruskal-Wallis $\mathrm{H}$ or ANOVA. The $\chi^{2}$-test for goodness of fit was used to verify agreement with Hardy-Weinberg equilibrium. Cumulative survival plots according to the genotype were evaluated using univariately by Kaplan-Meier analysis (log-rank test). Multivariate logistic analysis was performed to determine independent factors for occurrence of MI. A 2-sided probability level of $\leq 0.05$ was used for statistical significance. All analyses were done with SPSS for Windows 13.0 (SPSS Inc, Chicago, IL, USA).

\section{Results}

The genotype distribution in patients with $\mathrm{MI}$ and controls was in Hardy-Weinberg equilibrium (all $\mathrm{p}>0.05)$. In this study, we performed rs1333049 and rs10757274 genotypes in 250 control subjects and found they were in strong linkage disequilibrium $\left(r^{2}=0.92\right)$. Therefore, $r s 1333049$ was finally chosen in the study.

Clinical characteristics of the controls and patients with $\mathrm{Ml}$ are shown in Table 1. Compared with controls, patients with $\mathrm{Ml}$ had more traditional risk factors with higher fasting glucose, apoB, lipoprotein (a), and creatinine concentrations and lower apoA or HDL-C level (all $p<0.01$ ). No association of conventional risk factors with rs1333049 was observed in patients or controls.

Patients with $\mathrm{MI}$ had higher frequencies of the CC genotype $(30.0 \%$ vs. $20.7 \%)$ or $C$ allele $(55.5 \%$ vs. $46.2 \%$ ) compared with controls (all $p<0.01$ ) using univariate analysis (Table 1). Multivariate logistic regression analysis revealed that genotypes carrying allele $C$ were independent risk factors for $\mathrm{Ml}$ after adjustment for conventional risk factors [odds ratio (OR) 1.41, 95\% confidence interval (Cl) 1.17-1.70, $\mathrm{p}<0.001]$. Body mass index (BMI), triglyceride, total cholesterol, low HDL-C, lipoprotein (a), and creatinine were also found to be independent determinants for MI (Table 2).

There was no significant difference in number of diseased coronary arteries, lesion morphology, infarct site, and stroke among the subgroups of rs1333049 genotype (Table 3). Also there was no difference in LVEF, incidence of recurrence of non-fatal MI, recurrent angina, heart failure requiring hospitalization or composite MACE among patients with varying genotypes (Table 4). Cumulative survival rate was also similar among the three genotypes (Figure 1).

\section{Discussion}

The present study demonstrated that rs1333049 polymorphism on chromosome 9p21 was associated with $\mathrm{MI}$, but not with angiographic severity of CAD and 2 years occurrence of MACE after MI in Han Chinese.

The Wellcome Trust Case Control Consortium identified an association of rs1333049 polymorphism with CAD $\left(p<10^{-14}\right)$ in Caucasians (6). A later metaanalysis revealed that genotypes carrying allele $C$ were associated with an increase of $24 \% \quad 195 \%$ $\mathrm{Cl}=1.20-1.29$ ) in risk for CAD. Our results showed that the OR of allele $C$ for $\mathrm{Ml}$ was $1.41(95 \% \mathrm{Cl}$ 1.17-1.70) after adjusting for conventional risk 


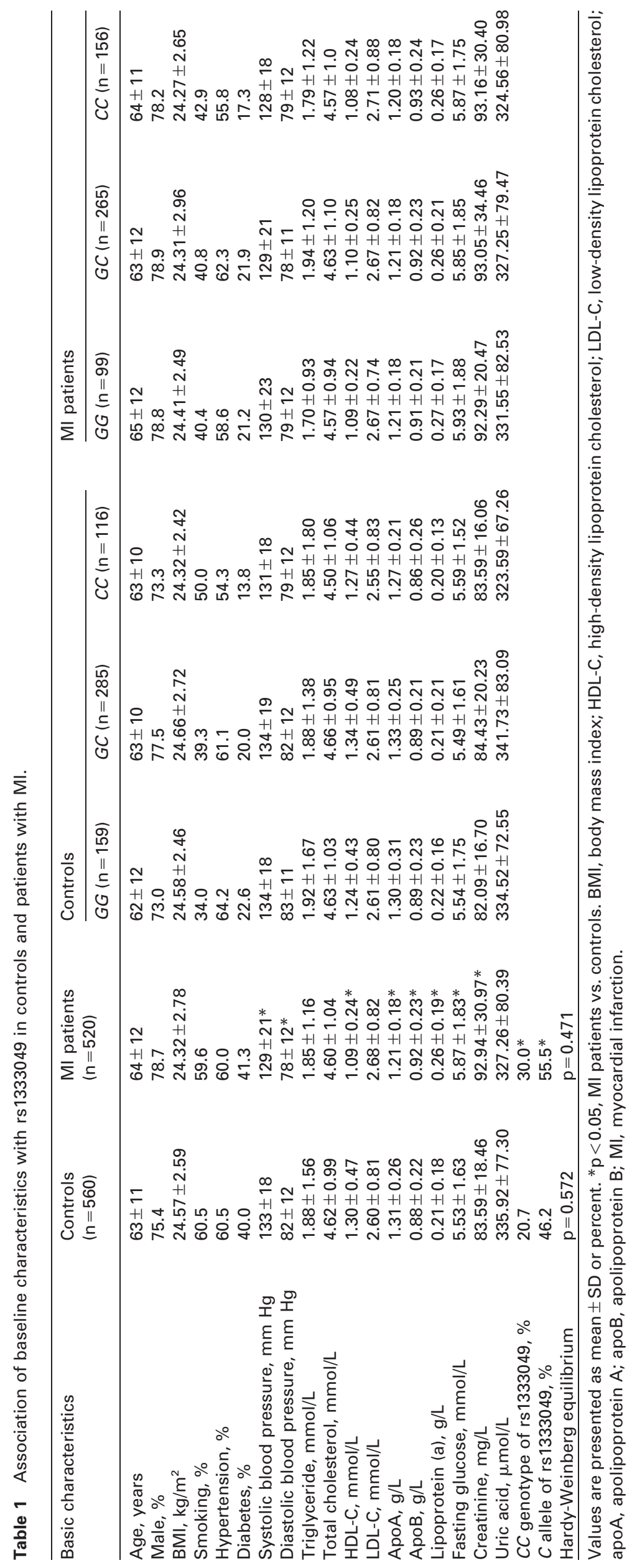


Table 2 Multivariate logistic regression analysis.

\begin{tabular}{llrl}
\hline Risk factors & OR & $\mathrm{p}$-Value & \multicolumn{1}{c}{$95 \% \mathrm{Cl}$} \\
\hline BMI & 0.93 & 0.006 & $0.89-0.98$ \\
Triglyceride & 0.85 & 0.020 & $0.75-0.98$ \\
Total cholesterol & 1.43 & 0.033 & $1.03-1.98$ \\
HDL-C & 0.08 & $<0.001$ & $0.05-0.15$ \\
LDL-C & 0.86 & 0.432 & $0.60-1.25$ \\
Lipoprotein (a) & 4.11 & $<0.001$ & $1.91-8.83$ \\
Fasting glucose & 1.04 & 0.281 & $0.97-1.12$ \\
Creatinine & 1.02 & $<0.001$ & $1.01-1.02$ \\
rs1333049 C allele & 1.41 & $<0.001$ & $1.17-1.70$ \\
\hline
\end{tabular}

$\mathrm{OR}$, odds ratio; $\mathrm{Cl}$, confidence interval; $\mathrm{BMI}$, body mass index; HDL-C, high-density lipoprotein cholesterol; LDL-C, low-density lipoprotein cholesterol; MI, myocardial infarction.

factors, indicating the possible impact of rs1333049 polymorphism on CAD in Chinese.

In this study, rs1333049 polymorphism was not associated with total number of diseased vessels or morphology of lesions. These observations were in line with the report by Anderson et al. (12), and suggested that variants at the $9 \mathrm{p} 21$ locus may robustly predict the prevalence of angiographic CAD, but not angiographic CAD severity. Assimes et al. revealed that chromosome 9p21 variation was associated with total coronary plaque burden as expressed by calcification scores (13). However, angiographic CAD severity defined as number of stenotic coronary arteries appeared to correlate weakly with atherosclerotic plaque burden of the coronary tree.

Several prospective studies have shown that chromosome 9p21 variation was associated with development of atherosclerosis and incident cardiovascular disease (14-16). However, Chen et al. revealed that $9 \mathrm{p} 21$ conferred null effects on the severity or progression of coronary atherosclerosis in Caucasians (17). Likewise, a recent report from the Rotterdam cohort study did not show an association of chromosome $9 \mathrm{p} 21$ with incidence of CAD or $\mathrm{MI}$ in older individuals (18). In our study, no association was observed between genotypes of rs1333049 polymorphism and occurrence of MACE after MI. These results suggest that the predictive value of chromosome 9 p21 for clinical outcomes in these patients may not be sufficient, at least for Han Chinese. This

Table 3 Association of rs 1333049 with severity of $\mathrm{MI}$ at baseline.

\begin{tabular}{|c|c|c|c|c|}
\hline & $G G(\mathrm{n}=99)$ & $G C(n=265)$ & $C C(\mathrm{n}=156)$ & $\mathrm{p}$-Value \\
\hline \multicolumn{5}{|l|}{ Number of diseased arteries } \\
\hline$\leq 1$ vessel disease, $\%$ & 42.4 & 37.7 & 32.1 & \\
\hline 2 vessel disease, \% & 32.3 & 34.3 & 36.5 & 0.550 \\
\hline 3 vessel disease, \% & 25.3 & 27.9 & 31.5 & \\
\hline Number of significant stenoses, $\mathrm{n}$ & 120 & 308 & 185 & 0.322 \\
\hline \multicolumn{5}{|l|}{ Morphology of significant lesions } \\
\hline Tyре A, \% & 12.5 & 17.9 & 14.1 & \\
\hline Type B1, \% & 31.7 & 33.8 & 30.3 & \\
\hline Type B2, \% & 37.5 & 35.9 & 34.6 & 0.221 \\
\hline Type C, \% & 18.3 & 12.4 & 21.1 & \\
\hline \multicolumn{5}{|l|}{ Echocardiography } \\
\hline LVEF, \% & $56.3 \pm 9.1$ & $57.5 \pm 8.1$ & $56.5 \pm 9.3$ & 0.608 \\
\hline \multicolumn{5}{|l|}{ Infarct site } \\
\hline Anterior MI, \% & 34.3 & 35.8 & 34.0 & 0.916 \\
\hline Inferior MI, \% & 48.5 & 40.8 & 41.0 & 0.384 \\
\hline Anterior+inferior MI, \% & 12.1 & 17.0 & 16.7 & 0.447 \\
\hline Length of hospital stay, days & $14 \pm 5$ & $14 \pm 4$ & $15 \pm 6$ & 0.015 \\
\hline Anti-hypertension treatment, \% & 31.4 & 24.7 & 24.6 & 0.387 \\
\hline Statin treatment, \% & 5.9 & 9.5 & 9.1 & 0.536 \\
\hline Ischemic stroke history, \% & 13.1 & 11.3 & 10.3 & 0.780 \\
\hline
\end{tabular}

Data are presented as mean \pm SD or percent. LVEF, left ventricular ejection fraction; MI, myocardial infarction.

Table 4 Association of rs1333049 with clinical outcome after MI.

\begin{tabular}{lllll}
\hline & $G G(\mathrm{n}=99)$ & $G C(\mathrm{n}=265)$ & $C C(\mathrm{n}=156)$ & $\mathrm{p}$-Value \\
\hline Treatment & & & & \\
$\quad$ Primary PCl, \% & 78.8 & 80.0 & 85.3 & 0.314 \\
Aspirin, \% & 90.9 & 94.3 & 91.7 & 0.410 \\
ACEl or ARB, \% & 67.7 & 64.9 & 62.8 & 0.730 \\
$\quad \begin{array}{l}\text { Blocker, \% } \\
\text { Statins, \% }\end{array}$ & 45.5 & 49.1 & 48.7 & 0.822 \\
Duration, months & 54.5 & 56.2 & 51.6 & 0.657 \\
Adverse events & $28 \pm 18$ & $30 \pm 17$ & $29 \pm 17$ & 0.685 \\
$\quad$ Rehospitalization, $\mathrm{n}$ & $1 \pm 1$ & $1 \pm 1$ & $1 \pm 1$ & 0.263 \\
$\quad$ Death, \% & 2.0 & 1.1 & 2.6 & 0.537 \\
$\quad$ Non-fatal MI, \% & 5.1 & 1.9 & 3.2 & 0.265 \\
$\quad$ Combined MACE, \% & 48.8 & 49.7 & 51.2 & 0.940 \\
\hline
\end{tabular}

Data are presented as mean \pm SD or percent. ACEI, angiotensin converting enzyme inhibitor; ARB, angiotensin II receptor blocker; MACE, major adverse cardiac events; MI, myocardial infarction; $\mathrm{PCl}$, percutanous coronary intervention. 


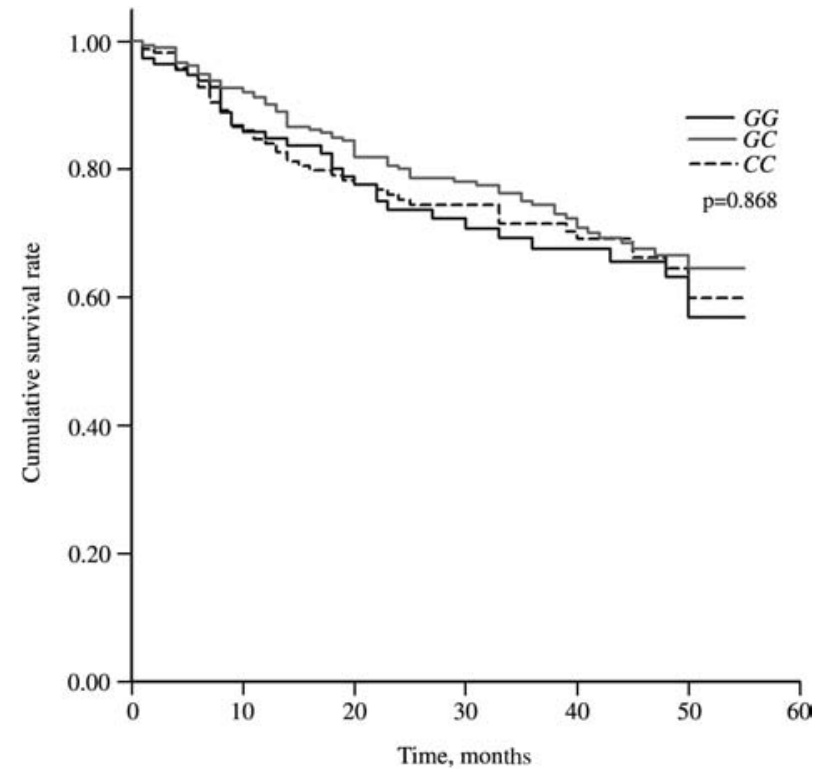

Figure 1 Kaplan-Meier survival plot by rs1333049.

Cumulative survival rate was similar among the $G G(n=99)$, $G C(n=265)$, and $C C(n=156)$ genotypes.

may be explained by the lack of relation between chromosome 9p21 polymorphism and left ventricular function and severity of CAD; the latter two factors were major determinants for prognosis in patients with MI. Furthermore, medical or interventional therapy could mitigate the influence of certain genetic factors including rs1333049 polymorphism in cardiovascular disease. Finally, to a certain extent, optimizing lifestyle and proper control of risk factors would weaken the genetic impact on atherosclerosis at the individual level.

In multiple logistic regression analysis, we found that serum creatinine was associated with $\mathrm{Ml}$ in addition to rs1333049. Renal impairment may be a marker for undiagnosed vascular disease or severity of vascular damage. It is also possible that atherogenic effects of traditional risk factors such as tobacco use, hyperlipidemia, hypertension, and diabetes may be greater in the presence of moderate to severe renal failure, which could also contribute to an increase in progression of CAD, MI and late mortality (19).

Some studies have probed the mechanisms behind the associative effect of chromosome 9 p21. The locus on chromosome 9p21 spans about 50-60 kb, containing no protein coding gene. Nevertheless, existing nearby is a cluster cell cycle promoting factor genes including cyclin-dependent kinase inhibitors $2 \mathrm{~A}$ and 2B (CDKN2A and $C D K N 2 B$ ). These play a significant role in regulating cell proliferation, senescence and apoptosis (20). It is possible that certain genetic variants in this region relate to the altered expression of $C D K N 2 A, C D K N 2 B$, and/or other genes located nearby $(21,22)$.

\section{Conclusions}

In summary, the rs1333049 polymorphisms on chromosome 9 p21 were associated with $\mathrm{Ml}$, but not angiographic severity of CAD and did not affect MACE rate during a 2-year follow-up in Han Chinese. Further large studies are needed to clarify the role of the locus at chromosome $9 \mathrm{p} 21$ in atherosclerosis and its complications.

\section{Conflicts of interest}

We did not accept any funding or support from an organization that may in any way gain or lose financially from the results of our study or the conclusions of our review. We are not employed by an organization that may gain or lose financially from the results of our study or our conclusions. We have no other conflicts of interest.

\section{Acknowledgements}

This study was supported by a key grant for Shanghai Science and Technology Foundation (05DZ19503).

\section{References}

1. Wang $\mathrm{Q}$. Molecular genetics of coronary artery disease. Curr Opin Cardiol 2005;20:182-8.

2. Ashavaid TF, Shalia KK, Kondkar AA, Todur SP, Nair KG, Nair SR. Gene polymorphism and coronary risk factors in Indian population. Clin Chem Lab Med 2002;40:97585.

3. Helgadottir A, Thorleifsson G, Manolescu A, Gretarsdottir S, Blondal T, Jonasdottir A, et al. A common variant on chromosome 9p21 affects the risk of myocardial infarction. Science 2007;316:1491-3.

4. Samani NJ, Erdmann J, Hall AS, Hengstenberg C, Mangino M, Mayer B, et al. Genomewide association analysis of coronary artery disease. N Engl J Med 2007;357: 443-53.

5. McPherson R, Pertsemlidis A, Kavaslar N, Stewart A, Roberts R, Cox DR, et al. A common allele on chromosome 9 associated with coronary heart disease. Science 2007;316:1488-91.

6. Consortium. WTCC. Genome-wide association study of 14,000 cases of seven common diseases and 3,000 shared controls. Nature 2007;447:661-78.

7. Schunkert $H$, Gotz A, Braund $P$, McGinnis R, Tregouet $\mathrm{DA}$, Mangino $\mathrm{M}$, et al. Repeated replication and a prospective meta-analysis of the association between chromosome $9 \mathrm{p} 21.3$ and coronary artery disease. Circulation 2008;117:1675-84.

8. Matarin M, Brown WM, Singleton A, Hardy JA, Meschia $\mathrm{JF}$. Whole genome analyses suggest ischemic stroke and heart disease share an association with polymorphisms on chromosome 9p21. Stroke 2008;39:1586-9.

9. Helgadottir A, Thorleifsson G, Magnusson KP, Gretarsdottir S, Steinthorsdottir V, Manolescu A, et al. The same sequence variant on 9 p21 associates with myocardial infarction, abdominal aortic aneurysm and intracranial aneurysm. Nat Genet 2008;40:217-24.

10. Ye S, Willeit J, Kronenberg F, Xu Q, Kiechl S. Association of genetic variation on chromosome 9p21 with susceptibility and progression of atherosclerosis: a populationbased, prospective study. J Am Coll Cardiol 2008;52: 378-84.

11. Ellis SG, Vandormael MG, Cowley MJ, DiSciascio G, Deligonul U, Topol EJ, et al. Coronary morphologic and clinical determinants of procedural outcome with angio- 
plasty for multivessel coronary disease. Implications for patient selection. Multivessel Angioplasty Prognosis Study Group. Circulation 1990;82:1193-202.

12. Anderson JL, Horne BD, Kolek MJ, Muhlestein JB, Mower CP, Park JJ, et al. Genetic variation at the 9p21 locus predicts angiographic coronary artery disease prevalence but not extent and has clinical utility. Am Heart J 2008;156:1155-1162 e2.

13. Assimes TL, Knowles JW, Basu A, Iribarren C, Southwick A, Tang $\mathrm{H}$, et al. Susceptibility locus for clinical and subclinical coronary artery disease at chromosome 9p21 in the multi-ethnic ADVANCE study. Hum Mol Genet 2008; 17:2320-8.

14. Talmud PJ, Cooper JA, Palmen J, Lovering R, Drenos F, Hingorani $A D$, et al. Chromosome 9p21.3 coronary heart disease locus genotype and prospective risk of $\mathrm{CHD}$ in healthy middle-aged men. Clin Chem 2008;54:467-74.

15. Samani NJ, Raitakari OT, Sipila K, Tobin MD, Schunkert $\mathrm{H}$, Juonala $\mathrm{M}$, et al. Coronary artery disease-associated locus on chromosome 9p21 and early markers of atherosclerosis. Arterioscler Thromb Vasc Biol 2008;28: 1679-83.

16. Doria A, Wojcik J, Xu R, Gervino EV, Hauser TH, Johnstone $\mathrm{MT}$, et al. Interaction between poor glycemic control and 9p21 locus on risk of coronary artery disease in type 2 diabetes. J Am Med Assoc 2008;300:2389-97.

17. Chen SN, Ballantyne CM, Gotto AM Jr, Marian AJ. The 9p21 susceptibility locus for coronary artery disease and the severity of coronary atherosclerosis. BMC Cardiovasc Disord 2009;9:3.

18. Dehghan A, van Hoek M, Sijbrands EJ, Oostra BA, Hofman A, van Duijn C, et al. Lack of association of two common polymorphisms on $9 \mathrm{p} 21$ with risk of coronary heart disease and myocardial infarction; results from a prospective cohort study. BMC Med 2008;6:30.

19. Zhang RY, Zhu ZB, Zhang Q, Yang ZK, Hu J, Lv AK, et al. Impact of moderate or severe renal insufficiency on long-term outcomes in patients undergoing drug-eluting stent based coronary intervention. Int J Cardiol 2008; Jul 22 [Epub ahead of print].

20. Gil J, Peters G. Regulation of the INK4b-ARF-INK4a tumour suppressor locus: all for one or one for all. Nat Rev Mol Cell Biol 2006;7:667-77.

21. Pasmant E, Laurendeau I, Heron D, Vidaud M, Vidaud D, Bieche I. Characterization of a germ-line deletion, including the entire INK4/ARF locus, in a melanoma-neural system tumor family: identification of ANRIL, an antisense non-coding RNA whose expression coclusters with ARF. Cancer Res 2007;67:3963-9.

22. Broadbent HM, Peden JF, Lorkowski S, Goel A, Ongen $H$, Green $F$, et al. Susceptibility to coronary artery disease and diabetes is encoded by distinct, tightly linked SNPs in the ANRIL locus on chromosome 9p. Hum Mol Genet 2008;17:806-14. 